\title{
Microscopic Characterization of CIS/CIGS Nanoparticles Prepared by Reactive Mechanical Milling
}

\author{
I. Santana-García ${ }^{1}$, C. Song ${ }^{2}$, C.F. Kisielowski ${ }^{2}$ and H. A. Calderon ${ }^{1}$ \\ 1. Dept. Física, ESFM-IPN, Mexico D.F. 07338, Mexico \\ 2. NCEM-JCAP, LBNL, Berkeley, CA, USA
}

Nanoparticles with photovoltaic (PV) properties can be used for increasing the efficiency of light capturing devices. CIS (CuInSe $)$ and CIGS $\left(\mathrm{CuIn}_{1-\mathrm{x}} \mathrm{Ga}_{\mathrm{x}} \mathrm{Se}_{2}\right)$ are good materials to use in developing a nanoPV process. CIS and CIGS have yielded thin-film solar cells with around $20 \%$ light conversion [1]. They contain no toxic elements and are highly absorbent in the visible light range. Additionally size quantization can increase the band gap of CIS from $1.0 \mathrm{eV}$ to $1.3 \mathrm{eV}$ with a corresponding better match to the solar spectrum. Small variations in composition can introduce $n$ - and $\mathrm{p}$ - doping and in the case of CIGS there is a possibility to do band gap engineering by growing core-shell structures. In this investigation a simple technique is used to synthesize nanoparticles in a dispersing medium. Reactive milling is used to chemically reduce chlorides of the involved elements with $\mathrm{Na}$. Such a chemical reaction produces a compound when the chlorides are added in stoichiometric proportions. Additionally $\mathrm{NaCl}$ (also a reaction product) is added from the beginning of milling in order to disperse the reaction products and allow synthesis of nanoparticles. Microscopic characterization is performed by using TEM in the TEAM 05 instrument. In all cases a low dose rate and an accelerating voltage of $80 \mathrm{keV}$ are employed in order to avoid a strong beam-sample interaction since these materials are highly sensitive.

Figure 1 shows X-ray diffraction patterns of as milled powders for three different systems $\mathrm{CuSe}_{2}$, $\mathrm{CuInSe}_{2}$ and $\mathrm{CuGaSe}_{2}$ ( preliminary for the synthesis of CIGS). In all cases diffraction peaks sharpen slightly as the milling time increases suggesting a gradual adjustment of the compound composition. Figure 2 shows an EDS global analysis that indicates a fair agreement with the stoichiometry of the compound (CIS).

Figure 3 shows CuInSe $\mathrm{C}_{2}$ nanoparticles after $5 \mathrm{~h}$ of milling. A total of 40 images are taken at different defoci to apply an Exit Wave Reconstruction (EWR) procedure by means of MacTempas (C). Two images are shown as examples in Fig. $3(\mathrm{a}-\mathrm{b})$, they are acquired by using an electron dose rate of $50 \mathrm{e}^{-}$ $/ \AA^{2}$ s. The resulting phase image is also given together with a colored image that clearly shows the structure and distribution of heavier atoms (In, see dots with higher intensity as pointed by arrow) in the expected lattice positions. This particle is still larger than desired but the processing can be adjusted to reduce particle size. Nevertheless, this analysis suggests that the stoichiometry of the compound is achieved and the procedure is effective to synthesize isolated nanoparticles instead of the massive powders that are commonly produced by mechanical milling.

Figure 4 shows representative images of $\mathrm{CuGaSe}_{2}$ particles after $7 \mathrm{~h}$ of mechanical milling. Three experimental images are shown from the focal series used to produce the phase image (Fig. 4c) by EWR. The image contrast is clearly increased after EWR which together with the low dose rate in use $\left(60 \mathrm{e}^{-} / \AA^{2} \mathrm{~s}\right)$ allows recording of the images. The beam sensitivity of particles containing Ga is extremely high and that accounts for the clear differences between the images in Figs. 3 and 4.

References.

[1] M. A. Green, Physica E 14 (2002) 11-17. 
[2] CONACYT, ICYT-DF (PICSO 11-24) and IPN are gratefully acknowledged for financial support through research grants. COFAA-IPN is also acknowledged for financial support.

[3] NCEM-LBL and DOE are gratefully acknowledged for the use of TEAM 05.

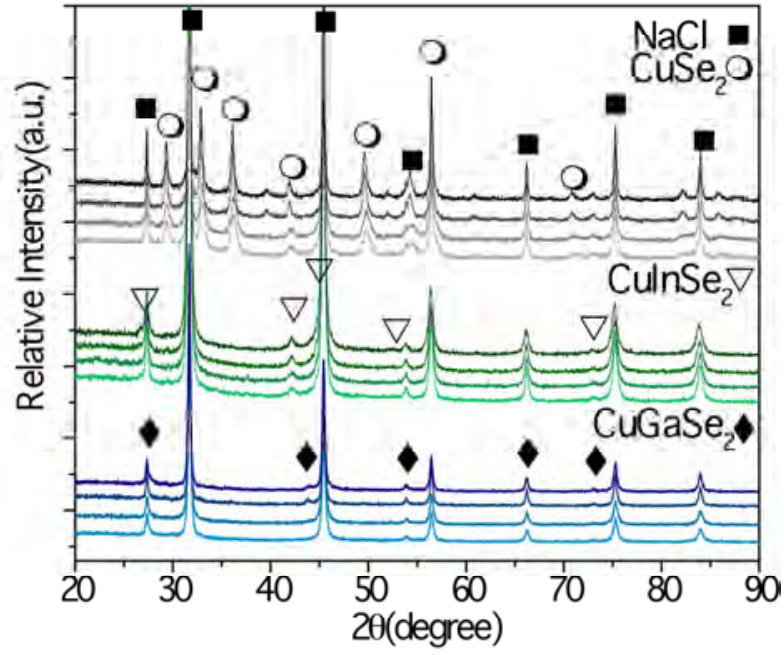

Figure 1. X-ray diffraction patterns of three systems for $3,5,7$ and $10 \mathrm{~h}$ of reactive milling.

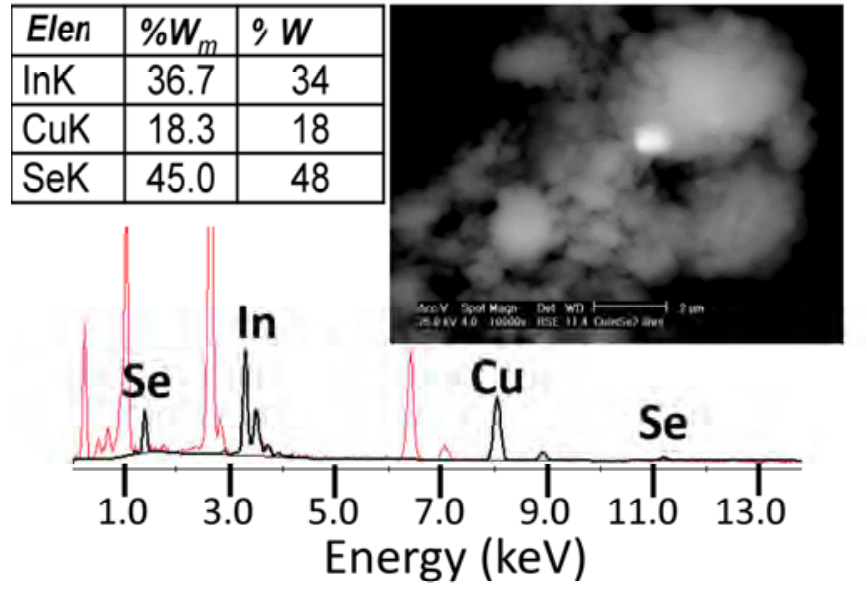

Figure 2. SEM image for $\mathrm{CuInSe}_{2}$ as-milled powders after $3 \mathrm{~h}$ of milling and corresponding EDS chemical analysis.
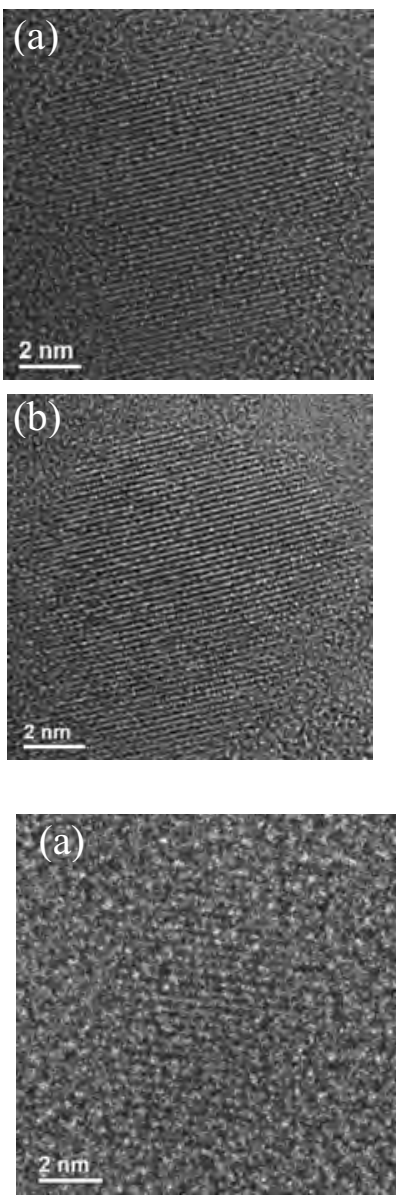
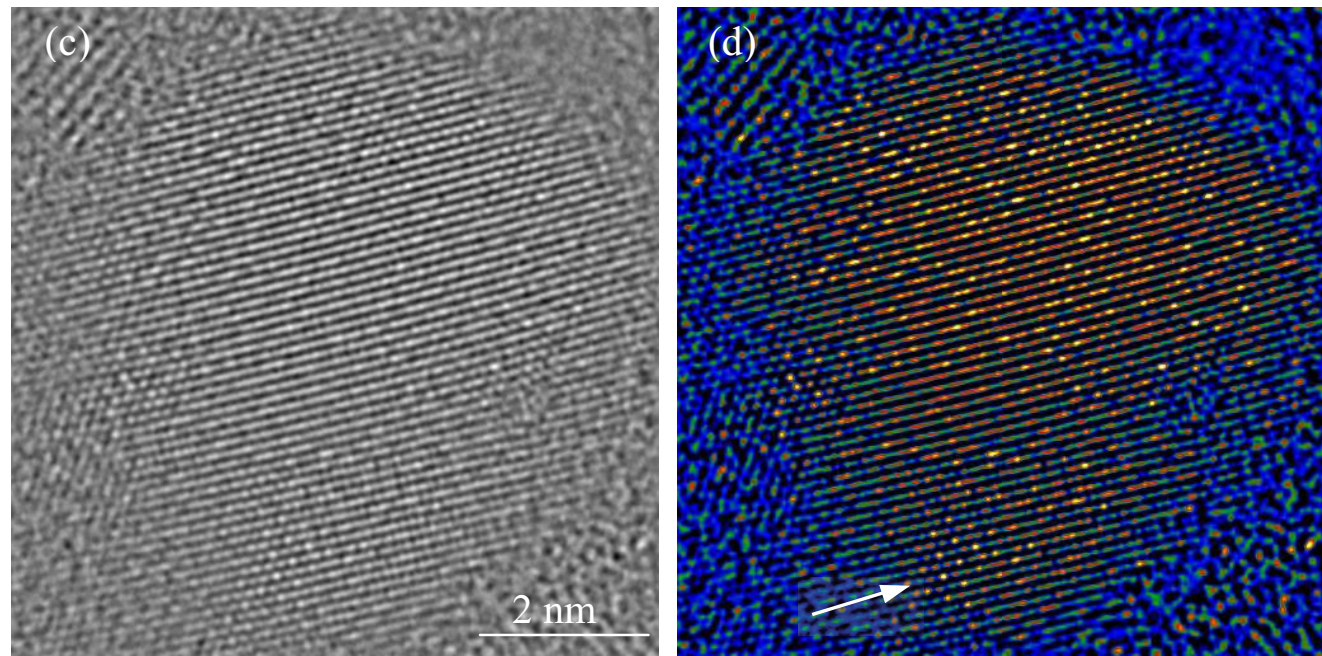

Figure 3. CuInSe $\mathrm{Se}_{2}$ nanoparticles. Experimental images at a defocus of (a) 220 $\mathrm{nm}$ and (b) $0 \mathrm{~nm}$ and taken with a dose rate of $50 \mathrm{e}^{-} / \AA^{2} \mathrm{~s}$. (c) Phase image after EWR and (d) in color. Arrow indicates atomic ordering in structure.
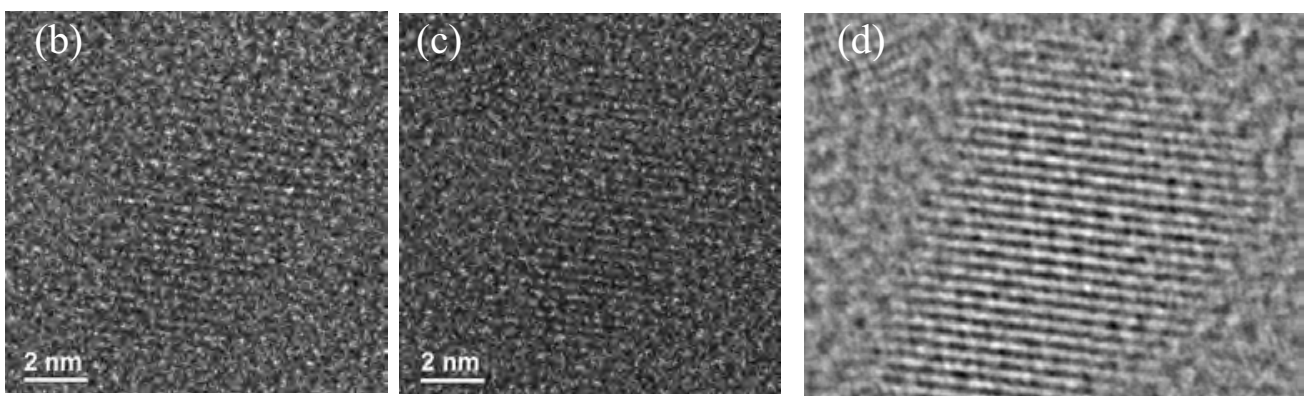

Figure 4. $\mathrm{CuGaSe}_{2}$ nanoparticle. (a-c) Experimental images at 165, 0 and $-165 \mathrm{~nm}$ of defocus. (c) Phase image after EWR. 